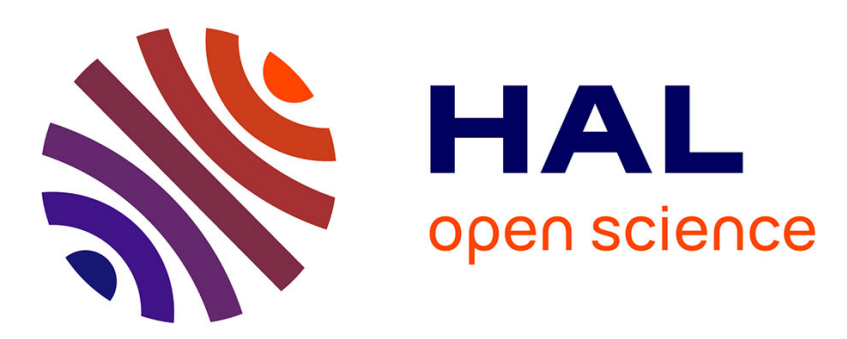

\title{
L'abbatiale carolingienne de Saint-Philbert-de-Grandlieu. Découvertes épigraphiques anciennes et récentes
}

Cécile Treffort

\section{- To cite this version:}

Cécile Treffort. L'abbatiale carolingienne de Saint-Philbert-de-Grandlieu. Découvertes épigraphiques anciennes et récentes. Bulletin Monumental, 2015, 173 (2), pp.151-156. 10.3406/bulmo.2015.12099 . halshs-01174744

\author{
HAL Id: halshs-01174744 \\ https://shs.hal.science/halshs-01174744
}

Submitted on 1 Jun 2020

HAL is a multi-disciplinary open access archive for the deposit and dissemination of scientific research documents, whether they are published or not. The documents may come from teaching and research institutions in France or abroad, or from public or private research centers.
L'archive ouverte pluridisciplinaire HAL, est destinée au dépôt et à la diffusion de documents scientifiques de niveau recherche, publiés ou non, émanant des établissements d'enseignement et de recherche français ou étrangers, des laboratoires publics ou privés. 


\section{L'abbatiale carolingienne de Saint-Philbert-de-Grandlieu.}

\section{Découvertes épigraphiques anciennes et récentes}

\section{Cécile Treffort}

\section{Citer ce document / Cite this document :}

Treffort Cécile. L'abbatiale carolingienne de Saint-Philbert-de-Grandlieu. Découvertes épigraphiques anciennes et récentes. In: Bulletin Monumental, tome 173, n², année 2015. pp. 151-156;

doi : https://doi.org/10.3406/bulmo.2015.12099

https://www.persee.fr/doc/bulmo_0007-473x_2015_num_173_2_12099

Fichier pdf généré le 29/10/2019 


\title{
Résumé
}

En 1896, trois inscriptions furent découvertes à Saint-Philbert-de-Grandlieu par le Père de la Croix : une dalle funéraire du XVe siècle et, surtout, une inscription de dédicace ainsi que l'épitaphe d'un moine nommé Guntarius, à associer sans aucun doute à l'ancienne abbatiale carolingienne. Depuis, d'autres signes graphiques, malheureusement encore non déchiffrés pour certains, ont été repérés sur les murs de l'édifice, donnant un éclairage particulier à son étude et à son histoire.

\begin{abstract}
The Carolingian abbatial church of Saint-Philbert-de-Grandlieu. Ancient and recent epigraphic discoveries

In 1896 thee inscriptions were discovered by Père de la Croix at Saint-Philbert-de-Grandlieu : a fifteenth-century funerary slab and, especially, a dedicatory inscription as well as the epitaph of a monk named Guntarius, quite certainly related to the Carolingian abbey church. Since then, the discovery of other graphic signs on the walls of the building, some of which have not yet been deciphered, have shed special light on the study of its history.
\end{abstract}

\section{Zusammenfassung}

Die karolingische Abteikirche Saint-Philibert-de-Grandlieu. Alte und neuere epigraphische Entdeckungen

Im Jahre 1896 entdeckte der belgische Archäologe Camille de la Croix in der Kirche Saint-Philibert drei Inschriften : eine Grabinschrift aus dem 15. Jh. und insbesondere eine Weiheinschrift, sowie die Grabinschrift eines Mönchs mit dem Namen Guntarius, der zweifellos mit der ehemaligen karolingischen Abteikirche verbunden war. Seitdem konnten an den Wänden des Bauwerks weitere Schriftzeichen identifiziert, leider aber nicht immer entziffert werden ; sie geben aber neue Impulse für Untersuchungen und die Geschichte der Anlage. 


\title{
L'ABBATIALE CAROLINGIENNE DE SAINT-PHILBERT-DE-GRANDLIEU
}

\section{DÉCOUVERTES ÉPIGRAPHIQUES ANCIENNES ET RÉCENTES}

\author{
Cécile TREFForT *
}

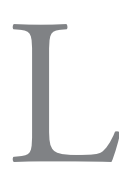

ors des travaux effectués en 1896 dans l'ancienne abbatiale de SaintPhilbert de Grandlieu, furent découvertes plusieurs inscriptions médiévales, dont une très belle pierre tombale du XV siècle ${ }^{1}$ (fig. 1). Moins monumentales mais plus intéressantes sans doute dans le cadre d'une étude sur l'édifice, deux autres inscriptions ont été dégagées dans ce qui était alors la chapelle Sainte-Anne, à l'est de la confessio : sur un des jambages d'un arc, une inscription de dédicace, mise au jour sous l'enduit ${ }^{2}$, et dans le sol, une épitaphe ${ }^{3}$. Enfin, à la croisée du transept, l'enlèvement du badigeon avait également fait apparaître divers éléments tracés sur la pierre, en particulier, du côté nord, quelques lettres dans un cercle formant une inscription assez énigmatique, signalées dès 1900 par P. de Berthou ${ }^{4}$, et du côté sud, quelques graffitis parmi lesquels on reconnaît le nom de Daniel, inédits jusqu'à la publication de Lebouteux en $1965^{5}$. Deux autres, découverts en 2015 , sont ici publiés pour la première fois ${ }^{6}$.

La plupart de ces inscriptions, considérées comme d'assez haute époque, étaient connues du Père de la Croix, y compris les deux situées dans le transept, dont on retrouve des estampages dans le fonds De la Croix déposé par la Société des antiquaires de l'Ouest aux Archives départementales de la Vienne à Poitiers ${ }^{7}$, même s'il ne les utilise pas dans ses publications. L'inscription de dédicace, l'épitaphe de Guntarius ${ }^{8}$ et les graffitis ont par ailleurs fait l'objet d'une notice détaillée dans le $23^{\mathrm{e}}$ volume du Corpus des inscriptions de la France médiévale ${ }^{9}$. La présente étude s'inscrit toutefois dans une perspective plus large, initiant une réflexion sur la place de ces traces épigraphiques dans la compréhension de l'histoire du monument ${ }^{10}$. Même si elles ne comportent ni millésime, ni référence à des évènements ou des personnages connus par ailleurs, leur rareté ${ }^{11}$ et, surtout, leur lieu de découverte en font des pièces archéologiques majeures.

\section{L'INSCRIPTION DE DÉDICACE}

Celle qui a suscité le plus d'intérêt est, comme on peut s'en douter, l'inscription de dédicace, qui a été considérée immédiatement comme celle de l'église. Découverte le 9 septembre 1896 et dessinée par le Père de la Croix dans l'un de ses carnets ${ }^{12}$, elle porte un texte très court ${ }^{13}$ :

\section{Idus Jun(ii) dedicatio $D($ e) i Salvatoris}

Aux ides de juin, dédicace au Dieu Sauveur.

Disposée sur quatre lignes sans cadre ni réglure, peu profondément gravée, elle présente une écriture assez soignée, malgré l'irrégularité de la taille des caractères (fig. 2). Dès sa découverte, elle a été associée par Léon Maître à la dédicace de l'église qui aurait suivi l'arrivée des reliques en 836 et les transformations de l'église qu'il estime terminées aux ides de juin suivantes, c'està-dire le 13 juin $837^{14}$. La paléographie de l'inscription incite toutefois à rester prudent à ce propos, car si l'usage exclusif de capitales romaines, même de module variable, peut s'accorder sans problème avec le IX ${ }^{\mathrm{e}}$ siècle, on ne peut pas exclure catégoriquement l'éventualité d'une datation plus tardive d'un ou deux siècles.

Une chose est cependant certaine : cette inscription se trouve à son emplacement d'origine, faisant corps avec son support, à savoir un pilastre supportant un arc donnant visuellement accès à la confessio. On peut évidemment regretter que le formulaire de cette inscription soit limité au jour de la cérémonie dédicatoire et au vocable, sans que soit mentionnée ni l'année, ni même la structure (autel, chapelle, église) concernée, comme c'est parfois le cas ${ }^{15}$. Toutefois, sa localisation et sa relative discrétion plaident pour l'associer plus volontiers à un autel particulier qu'à l'église toute entière. On connaît ainsi plusieurs cas où le texte commémorant la dédicace des autels latéraux est gravé à même les blocs formant pilastre à proximité immédiate. On peut se référer, par exemple, à l'église clunisienne de Saint-Jean de Montierneuf à Poitiers, pour la fin du XI ou le tout début du XII ${ }^{\mathrm{e}}$ siècle ${ }^{16}$, ou plus encore, au XI ${ }^{\mathrm{e}}$ siècle, à la petite église de Peyrusse-Grande, dans le Gers ${ }^{17}$, dont les inscriptions de dédicace des deux autels latéraux Saint-Martin et Saint-Jean utilisent une formule similaire à celle de SaintPhilbert. L'indication limitée au jour de la cérémonie et au vocable peut être encore plus discrète, directement portée sur le pied de l'autel, comme c'était vraisemblablement le cas à Quarante, dans l'Hérault ${ }^{18}$.

À Saint-Philbert, le vocable, par ailleurs très carolingien, de Saint-Sauveur ${ }^{19}$, a pu être attribué non à l'abbatiale toute entière, mais plutôt à un autel installé à proximité. Si la date indiquée par l'inscription, le 13 


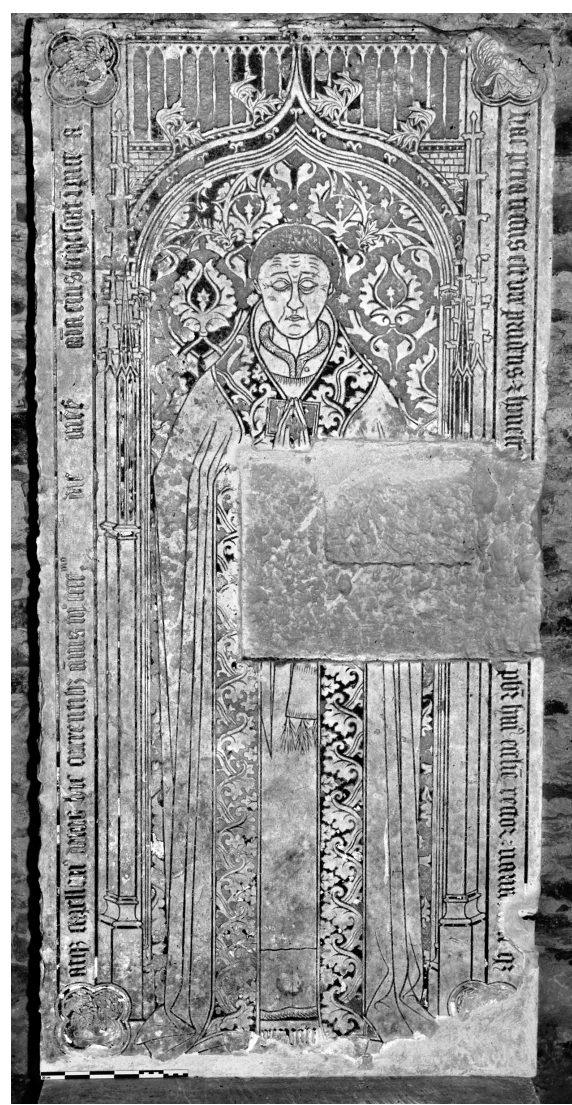

Cl. J.-P. Brouard / CIFM

Fig. 1 - Saint-Philbert-de-Grandlieu, abbatiale, pierre tombale $\mathrm{du} \mathrm{XV}^{\mathrm{e}}$ siècle, attribuée à Guillaume Chupin.

juin, ne correspond pas à la fête du saint (le 20 août ${ }^{20}$ ), elle est en revanche très proche de la date de l'élévation de ses reliques à Herio le 7 juin ${ }^{21}$ suivie de leur translation, et très proche voire identique à celle de leur dépôt, quelques jours plus tard, dans l'église de Deas ${ }^{22}$. Par la fusion liturgique ainsi créée, la superposition des deux dates (celle de la consécration de l'autel au Sauveur et celle de l'anniversaire de la translation des reliques du saint) pourrait parfaitement répondre à une certaine proximité topographique entre tombeau et autel. Si la date choisie pour la dédicace devait absolument tomber un dimanche, on pourrait alors resserrer encore la datation de l'évènement, puisqu'entre l'arrivée des reliques à Deas (en 836) et leur départ pour Cunault (en 858), il n'y a eu que quatre années où le 13 juin était un dimanche : 840, 846, 852 et $857^{23}$. Mais peut-être est-ce aller trop loin dans l'interprétation d'un texte épigraphique somme toute assez modeste.

\section{L'ÉPITAPHE DE GUNTARIUS}

La deuxième inscription découverte en 1896 à Saint-Philbert, l'épitaphe du moine Guntarius, provient à peu près du même endroit. Malheureusement, les conditions de sa découverte sont mal documentées ; à plusieurs reprises, Léon Maître la dit " trouvée en remuant les terres de cette crypte ${ }^{24}$. Cependant, il précise également dans une note : "On ne sait pas exactement si l'inscription est sortie de la crypte ou de la chapelle Sainte-Anne, qui ont été déblayées dans le même temps 25 ". Ce léger doute sur sa provenance exacte ne représente pas en soi un problème majeur, car il paraît évident qu'elle était déjà mutilée, et vraisemblablement extraite de son contexte primitif d'utilisation, lors de sa découverte, sur lequel il paraît donc difficile de disserter longuement. Exposée pendant longtemps contre un mur, enserrée dans un encadrement de bois qui ne laissait voir que sa face principale, elle a fait l'objet d'une restauration récente, qui lui a rendu toute sa beauté, et elle est aujourd'hui présentée dans l'abbatiale sous vitrine.

Il s'agit d'un bloc de pierre grossièrement parallélépipédique, avec en partie basse une sorte de biseau et une gorge aménagée sur toute sa largeur, dus soit à la réutilisation d'un bloc plus ancien, façonné pour un autre usage, soit à un aménagement spécifique lié à sa fonction funéraire

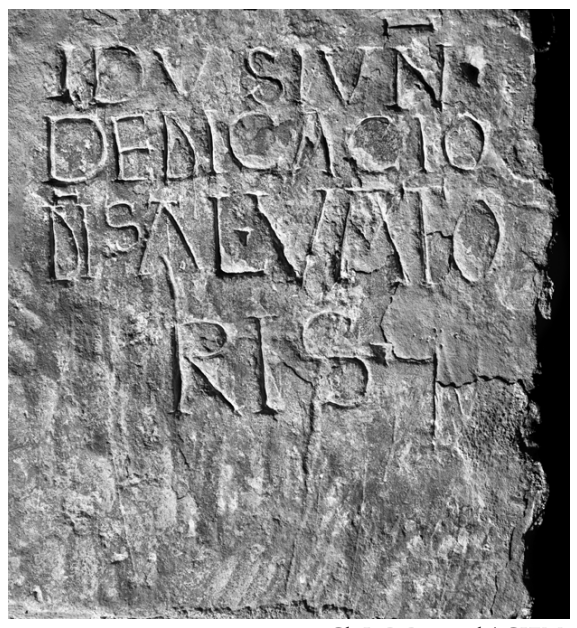

Cl. J.-P. Brouard / CIFM.

Fig. 2 - Saint-Philbert-de-Grandlieu, abbatiale, inscription de dédicace. (fig. 3). Initialement décorée par une croix latine en relief, bûchée ultérieurement, cette inscription relativement épaisse a pu être utilisée comme une stèle, fichée en terre à la verticale, plutôt que contre un mur. Si un tel dispositif est rare pour le haut Moyen Âge, il n'est pas inédit : on peut citer en particulier l'épitaphe d'Amalricus, datée du Xe siècle et découverte sur le site de Pouthumé, près de Châtellerault ${ }^{26}$, d'un module proche de celle de Saint-Philbert, ou, plus monumental, le pilier d'Estoublon, dans les Hautes-Alpes ${ }^{27}$. À Saint-Philbert, le texte, disposé dans les quatre angles formés par la sculpture de la croix, se lit section par section, d'abord à gauche, de haut en bas, puis de même à droite (fig. 4). Son contenu est réduit à une phrase ${ }^{28}$ :

Hic requiescit in tumulo / Guntarius nomine / mon[a]chus et [s]ace[rdos] qui / V id(us) jun(ii) obi[i]t in D(omi)no.

Ici repose dans ce tombeau Guntarius, moine et prêtre, qui mourut dans le Seigneur le 5 des ides de juin [9 juin].

Cette inscription a été réalisée avec grand soin dans un calcaire blanc; on perçoit encore à certains endroits les traits de réglure qui ont servi à composer le texte en belles capitales romaines, avec deux lettres anguleuses ( $\mathrm{G}$ carré, $\mathrm{O}$ en losange) et de nombreux enclavements et conjonctions. La paléographie et le formulaire utilisés s'accordent pour une attribution de cette inscription à l'époque carolingienne (IX $-X^{e}$ siècle) sans qu'il soit possible d'être plus précis.

On ignore malheureusement tout de ce Guntarius. On peut toutefois imaginer que pour avoir bénéficié d'une épitaphe aussi exceptionnelle pour l'époque ${ }^{29}$, il a dû jouer au sein de la communauté un rôle plus important que celui d'un simple moine, fût-il prêtre. La préservation de cette inscription, au texte bien conservé malgré la mutilation du décor, ne relève peut-être elle-même pas d'un simple hasard : pour qu'on la retrouve en cet endroit précis, sacralisé par la présence du tombeau du saint et, sinon par l'autel, du moins par le souvenir scriptural d'une dédicace au Sauveur, il a bien fallu qu'elle 


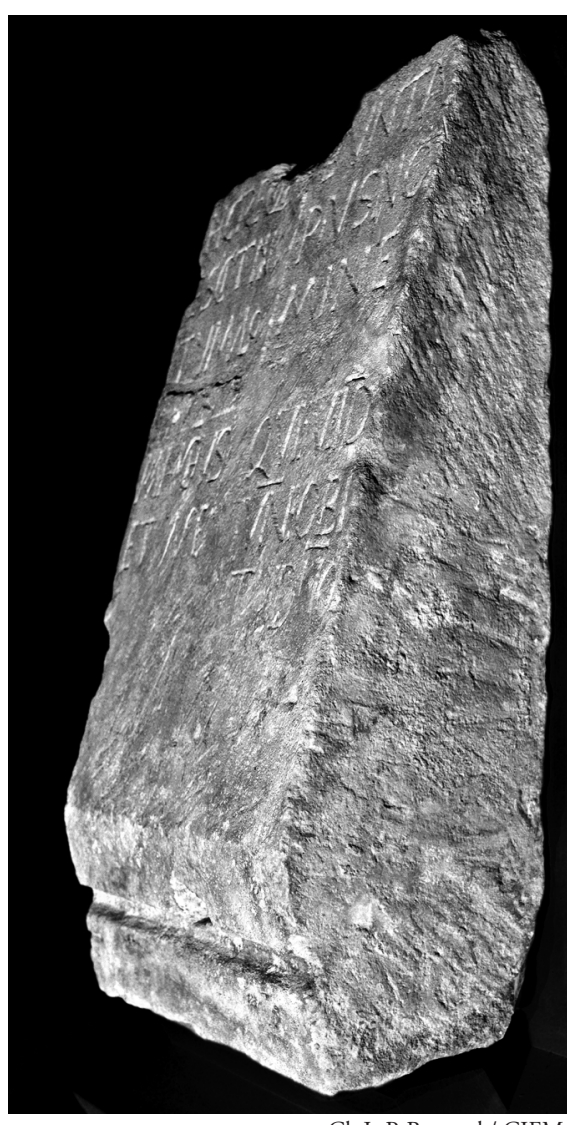

Cl. J.-P. Brouard / CIFM

Fig. 3 - Saint-Philbert-de-Grandlieu, abbatiale, épitaphe de Guntarius, vue de côté.

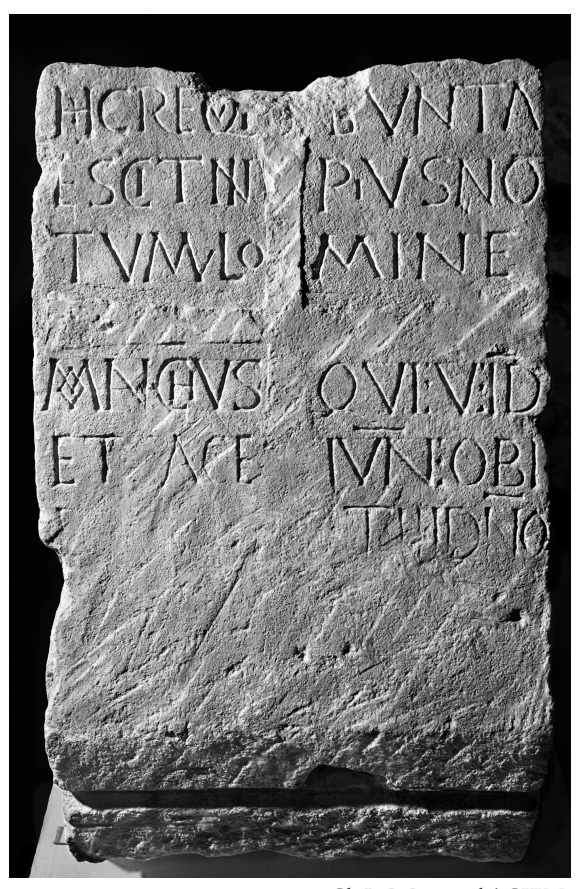

Cl. J.-P. Brouard / CIFM

Fig. 4 - Saint-Philbert-de-Grandlieu, abbatiale, épitaphe de Guntarius, vue de face. s'y trouve avant le remblaiement de l'espace. Soit elle y était originellement, associée à une sépulture privilégiée, soit on est allé la chercher, vraisemblablement à proximité, et sans aucun doute intentionnellement; dans un cas comme dans l'autre, la conservation de sa mémoire en ce lieu très spécial ressemble à un traitement de faveur accordé par la communauté de Deas à leur (vénéré ?) frère Guntarius.

\section{GRAFFITIS ET AUTRES SIGNES INSCRITS}

Pour terminer ce tour d'horizon épigraphique, il convient de signaler certaines traces inscrites, à la limite du graffiti, sur les arcs de la croisée du transept. Même si elles sont pour la plupart difficiles à lire ou à interpréter, elles n'en restent pas moins des témoignages précieux de la vie de l'édifice.

\section{Arc méridional de la croisée du transept}

Sur le pilier oriental de l'arc méridional (fig. 5), on trouve ainsi deux blocs portant des inscriptions, repérées lors de la mission 2015 sur leur face nord (côté nef).

Le plus haut des deux (A) porte un texte manifestement écrit en minuscule, avec des lettres hautes d'environ $1,5 \mathrm{~cm}$,

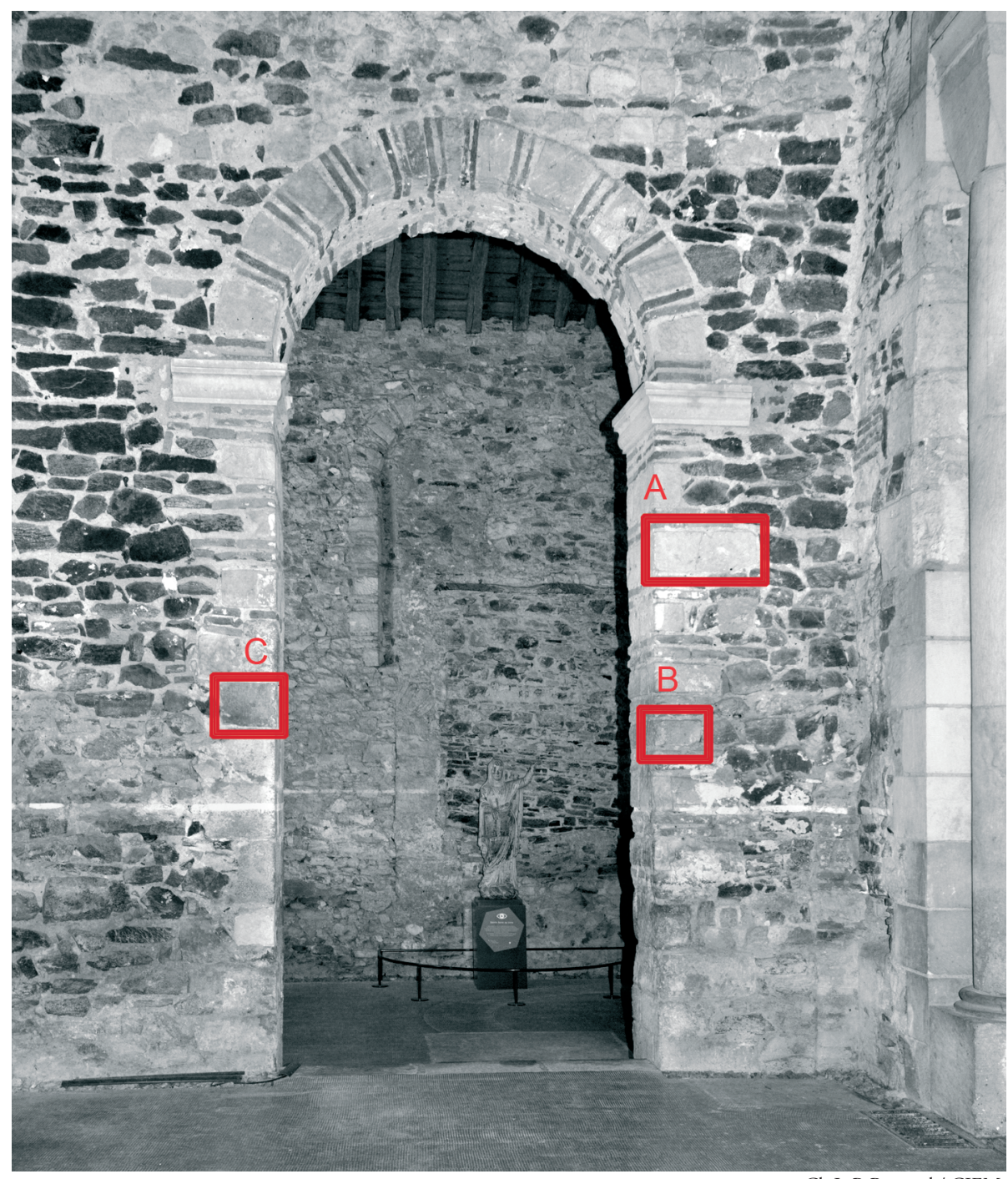

Cl. J.-P. Brouard / CIFM

Fig. 5 - Saint-Philbert-de-Grandlieu, abbatiale, arc méridional de la croisée du transept : localisation des blocs $\mathrm{A}, \mathrm{B}$ et $\mathrm{C}$. 


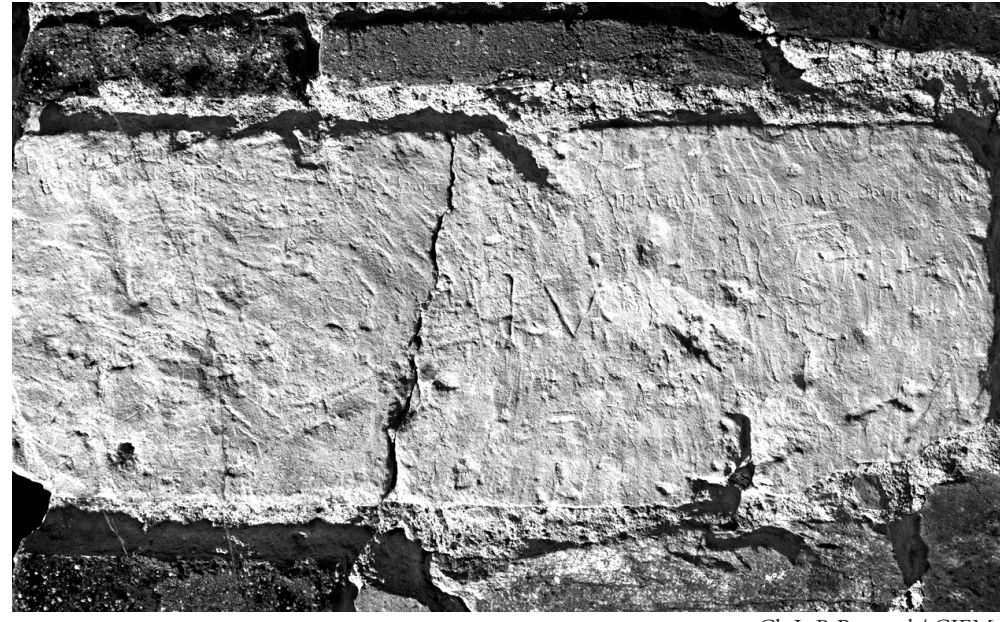

Cl. J.-P. Brouard / CIFM

Fig. 6 - Saint-Philbert-de-Grandlieu, abbatiale, inscription en minuscule sur la face nord du bloc $\mathrm{A}$.

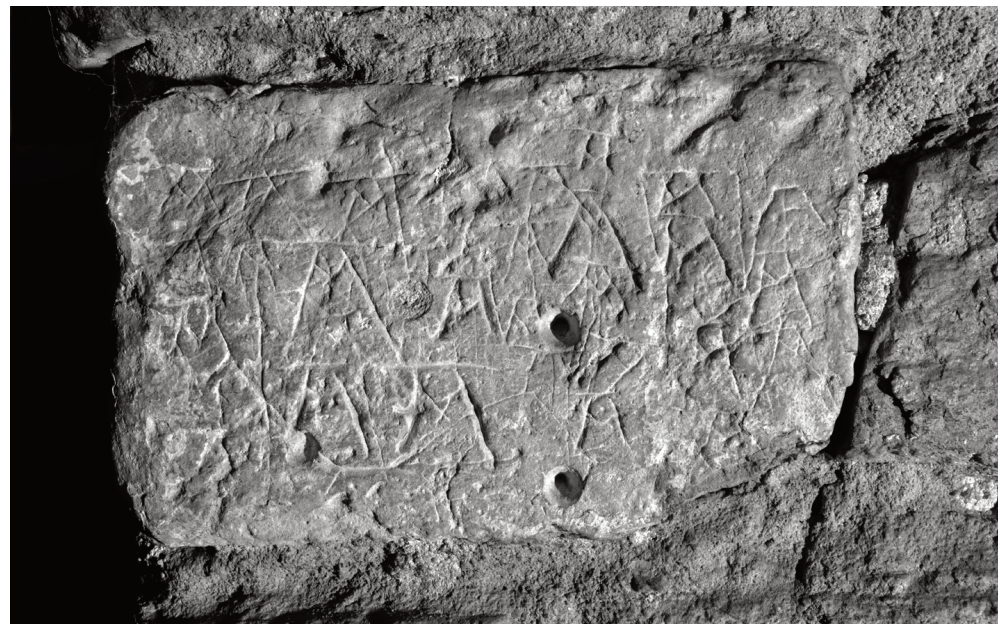

Cl. J.-P. Brouard / CIFM

Fig. 7 - Saint-Philbert-de-Grandlieu, abbatiale; série de A incisés sur la face nord du bloc B.

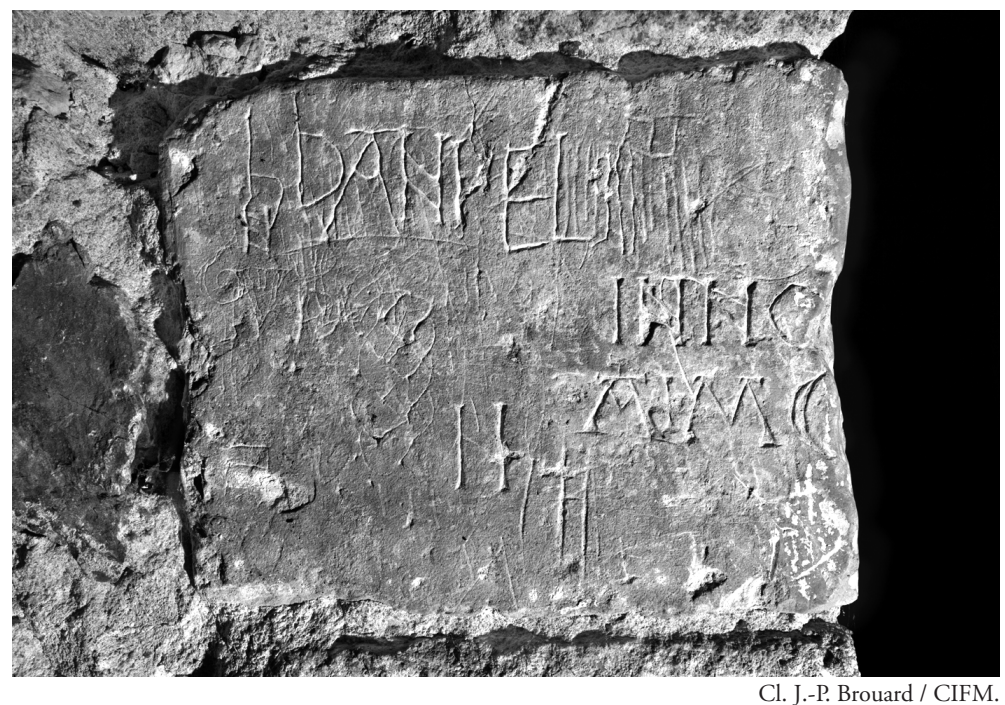

Fig. 8 - Saint-Philbert-de-Grandlieu, abbatiale, graffitis sur la face nord du bloc C. sur la largeur totale du bloc, soit une cinquantaine de centimètres (fig. 6). Encore rétif à toute tentative de lecture, ce texte laisse toutefois deviner, dans sa deuxième partie, le mot David, qui suggère qu'on pourrait avoir là une citation biblique, à moins qu'il ne s'agisse d'un nom propre médiéval.

Un peu plus bas, on trouve sur la surface entière d'un autre bloc (B) plus d'une vingtaine de lettres A incisées, certaines se recouvrant partiellement (fig. 7). La forme de ces $A$, à traverse brisée et plateau sommital, n'est pas sans rappeler l'un des A de l'inscription de dédicace, sans qu'il s'agisse toutefois d'un élément déterminant pour établir sans ambiguïté une relation entre les deux.

Sur un des blocs du pilier occidental du même arc $(\mathrm{C})$, toujours face nord (côté nef), on peut lire sur un bloc, parmi un lacis de graffitis, ces trois mots ${ }^{30}$ (fig. 8) :

\section{Daniel \\ In no[mine?] \\ Aimo}

Déjà connus du Père de la Croix ${ }^{31}$, ils sont attribuables, par leur paléographie (capitales romaines irrégulières, avec quelques barres transversales redoublées), à une période haute (IX ${ }^{\mathrm{e}}-\mathrm{X}^{\mathrm{e}}$ siècle), même s'il reste difficile d'en tirer des conclusions utiles pour la compréhension du site ${ }^{32}$.

\section{Arc septentrional de la croisée du transept}

Sur le pilier oriental de l'arc septentrional de la croisée du transept (fig. 9), faisant quasiment face aux précédentes, une inscription énigmatique associant une croix, deux cercles concentriques et quelques lettres profondément gravées, est visible sur la face sud - toujours côté nef d'un bloc (E) situé juste en dessous du tailloir (fig. 10). Connue par le Père de la Croix, qui en avait fait, ou fait faire, quelques estampages et frottis de carbone sur papier de soie ${ }^{33}$, et évoquée par P. de Berthou comme "inscription monogrammatique " ${ }^{34}$, elle garde pour l'instant tout 
son mystère, même si sa qualité, son emplacement et sa composition générale reflètent son importance.

Le bloc comporte, dans sa partie haute, un grand cercle de $12 \mathrm{~cm}$ de diamètre, avec en son centre un petit cercle de $4,5 \mathrm{~cm}$ de diamètre, partagé en quatre, et enfin un trou au milieu. Dans l'espace compris entre les deux cercles, on trouve une petite croix pattée en haut ; en tournant dans le sens des aiguilles d'une montre, on peut ensuite lire plusieurs lettres juxtaposées, qui ne forment toutefois pas un mot : peut-être les lettres VR VO VS, assez profondément gravées, puis une sorte de $\mathrm{D}$ oncial et peutêtre un E, simplement graffité. Pour l'instant, aucune lecture ni interprétation satisfaisante ne peut être proposée.
Cependant, le caractère monumental de l'inscription, son lien visuel direct avec le chœur et la présence d'une croix interdisent d'y voir un simple jeu : on penserait plutôt aux initiales des mots d'une formule qui nous échappe aujourd'hui ${ }^{35}$. À michemin entre une croix de consécration et la figuration d'une hostie, l'impression qui ressort de cet ensemble de signes est en tous cas celle d'une grande sacralité.

Enfin, sur le retour du bloc, côté ouest (face à l'intérieur de l'arc), parmi quelques traits incisés, on peut clairement lire Dani (fig. 11), sans doute le début du nom Daniel, mutilé par un éclat de pierre, sans qu'on puisse déterminer s'il s'agit ou non du même que celui qui se trouve sur l'arc opposé.

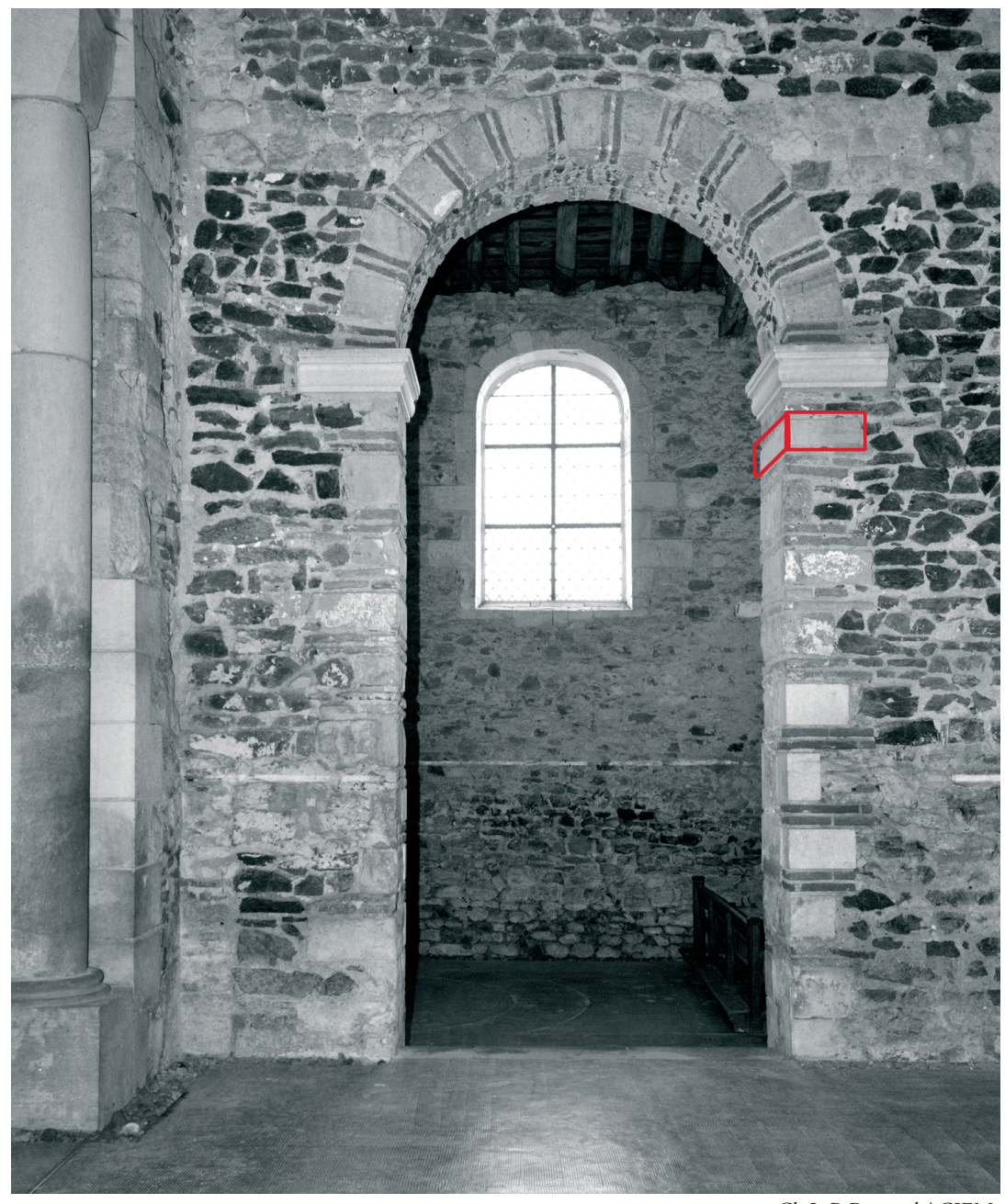

Cl. J.-P. Brouard / CIFM.

Fig. 9 - Saint-Philbert-de-Grandlieu, abbatiale, arc septentrional de la croisée du transept : localisation du bloc D.

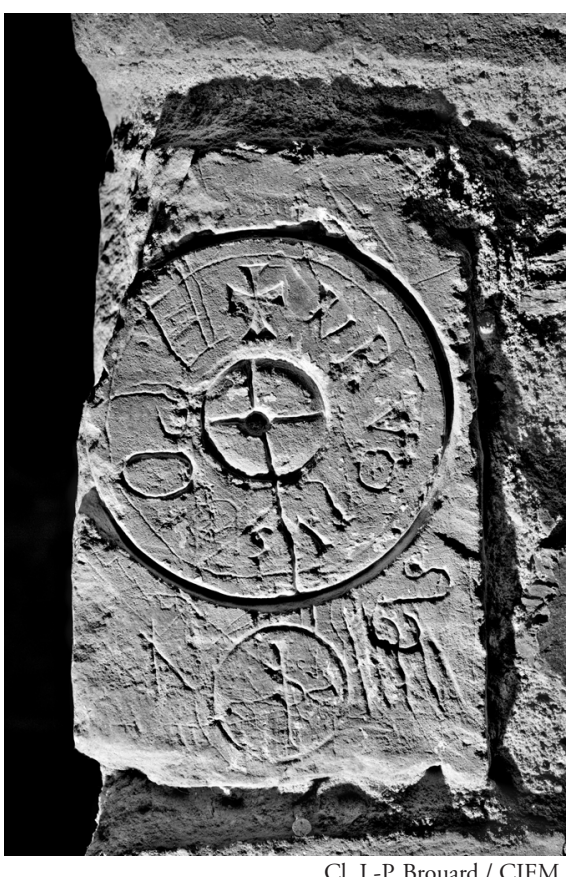

Fig. 10 - Saint-Philbert-de-Grandlieu, abbatiale, inscription énigmatique sur la face sud du bloc D.

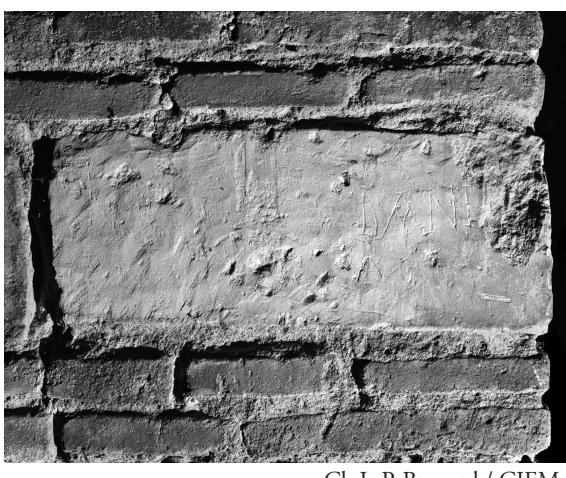

Cl. J.-P. Brouard / CIFM.

Fig. 11 - Saint-Philbert-de-Grandlieu, abbatiale, graffiti sur la face ouest du bloc D.

\section{CONCLUSION}

Si l'on excepte l'épitaphe de Guntarius, dont on ignore la localisation et la mise en scène initiales, toutes les traces épigraphiques inscrites ou incisées découvertes dans l'abbatiale de Saint-Philbert participent étroitement à l'histoire de l'édifice. Espérons que leur publication permettra à terme de résoudre les difficultés de lecture et d'interprétation qui persistent à ce jour, pour comprendre pleinement leur place et leur rôle dans cette histoire complexe mais passionnante. 


\section{CÉCILE TREFFORT}

\section{NoTES}

* Professeur en histoire médéviévale à l'université de Poitiers (CESCM)

1. Découverte mentionnée par Maître 1896 (1), p. 101 ; Maître 1898 (2), p. 182. Son texte est le suivant: [Sub] hac petra tectus est vir prudens (et) honestlus ...J p(res)b(i)t(e)r huj(us) eccle(es)ie nam[...]que|...] atq(ue) capellan(us) donec bic currenti(bus) a (n)nis M CCCC III die III me(n)s(is) III. $A(n) i(m)$ a ejus req[ui]escat $i(n)$ pace. $A(m e n)$. On remarquera que l'année précise, le jour et le mois n’ont pas été renseignés, l'espace réservé pour cela n'ayant jamais été gravé. Utilisée à partir de 1663 comme table d'autel, cette pierre tombale a été identifiée comme celle de Guillaume Chupin, recteur de Saint-Philbert, mort en 1449. Elle a été classée Monument historique au titre des Antiquités et objets d'art le 25 novembre 1896. Pour que ce tour d'horizon épigraphique soit complet, il convient de signaler enfin une petite inscription du début du XVII ${ }^{e}$ siècle sur un bloc formant, sur le mur gouttereau sud, le jambage gauche d'une petite porte, avec le texte suivant : Le 15 jui(n) 1602 fur cazee ceans.

2. Maître 1896 (1), p. 106, note 1 ; Maître 1898 (2), p. 202-203, note 2 .

3. Maître 1896 (1), p. 93 ; Maître 1898 (2), p. 195 196.

4. Bertou 1900, p. LIX.

5. Lebouteux 1965-1966, p. 83.

6. Découverte lors de la mission photographique complémentaire réalisée dans le cadre du Corpus des inscriptions de la France médiévale le 9 février 2015 avec Estelle Ingrand-Varenne et Jean-Pierre Brouard, que j'ai plaisir à remercier tout particulièrement pour ses beaux et précieux clichés. Je tiens également à exprimer ma gratitude à Mathilde Angelvy et Pauline Illegems-Martineau, qui ont grandement facilité mes recherches sur le site, ainsi qu’à François HéberSuffrin et Christian Sapin pour leur relecture du présent article.

7. Arch. dép. Vienne, cote générale $16 \mathrm{~J} 3$.

8. Qui toutes deux viennent de faire l'objet d'une restauration.

9. Corpus des inscriptions de la France médiévale, VIII XIII siècle, éd. V. Debiais, collab. R. Favreau, J. Michaud, C. Treffort, vol. 23, Côtes-d'Armor,
Finistère, Ille-et-Vilaine, Morbihan (région Bretagne), Loire-Atlantique et Vendée (région Pays de la Loire), Paris, 2008. Désormais CIFM.

10. On remarquera qu'une grande partie des publications concernant Saint-Philbert n'en fait même pas mention. Cf. par exemple Lasteyrie 1909, dont on connaît pourtant l'intérêt pour l'épigraphie.

11. G. Durville, Catalogue du musée archéologique de Nantes. 2ème partie : Musée lapidaire, Nantes, 1927, p. 98, rappelle que l'épitaphe de Guntarius est l'une des trois seules " inscriptions chrétiennes " antérieures à l'an mille conservées dans le département.

12. Arch. dép. Vienne, 16 J 3 / 60, p. 30.

13. CIFM, op. cit. note 9, 23, no 103, p. 106-107.

14. Maître 1896 (1), p. 106, note 1 ; Maître 1898 (2), p. 102-103, note 2 .

15. En attendant la thèse d'Annick Gagné en cours, on peut se reporter à la thèse de J. Michaud, Les inscriptions de consécration d'autels et de dédicace d'églises en France du VIII' au XIII' siècle. Épigraphie et liturgie, Th. $3^{e}$ cycle, dactyl., Poitiers, 1978.

16. CIFM, op. cit. note 9, I-1, n 72-75, p. 80-90.

17. CIFM, op. cit., note 9, 6, n 61-62, p. 66-67 et pl. XXII, fig. 42-43.

18. CIFM, op. cit. note $9,12, \mathrm{n}^{\circ}$ 50, p. 155-156. Considérée au moment de la publication comme perdue, cette inscription est en fait conservée au musée de Quarante, où elle a été vue et photographiée en 2002.

19. C. Heitz, Recherche sur les rapports entre architecture et liturgie à l'époque carolingienne, Paris, 1963, p. $145-163$.

20. Date anniversaire de sa mort à Herio, mentionnée à plusieurs reprises dans le dossier hagiographique : cf Ermentaire 1905 (Vita, 30 ; Miracula, I, 29 et 42 ; II, 11).

21. Ermentaire 1905 (Miracula, I, 2).

22. Les Miracula ne précisent pas la date de leur dépôt dans l'église de Deas ; Lasteyrie 1909, p. 4, propose la date du 11 janvier au terme d'un calcul fondé sur les indications hagiographiques, mais ces dernières ne semblent pas assez précises pour exclure une arrivée le 13 juin.
23. Calcul effectué à partir de Millesimo, outil calendaire dû à Denis Muzerelle, accessible en ligne (http://millesimo.irht.cnrs.fr).

24. Maître 1896 (1), p. 93 ; Maître 1898 (2), p. 195 196.

\section{Maître 1896 (1), p. 93, note 3.}

26. C. Treffort, M. Uberti, «Identité des défunts et statut du groupe dans les inscriptions funéraires des anciens diocèses de Poitiers, Saintes et Angoulême entre le IVe et $X^{e}$ siècle », L. Bourgeois éd., Wisigoths et Francs autour de la bataille de Vouillé (507). Recherches récentes sur le haut Moyen Âge dans le Centre-Ouest de la France. Actes des XVIII Journées internationales d'archéologie mérovingienne, Vouillé et Poitiers (Vienne, France), 28-30 septembre 2007, coll. «Mém. AFAM », Saint-Germain-en-Laye, 2010, fig. 15, p. 200.

27. Voir en dernier lieu P. Bogard éd., Estoublon, de la période antique aux temps médiévaux. Actes des rencontres d'Estoublon. Chronique de mille ans d'histoire. Estoublon, 8-9 octobre 2011, Valensole, 2012, p. 3136.

28. CIFM, op. cit., 23, nº 104, p. 107-108.

29. Pour une vue générale sur la question, voir C. Treffort, Mémoires carolingiennes. L'épitaphe entre genre littéraire, célébration mémorielle et manifeste politique (milieu VIII"-XI" siècle), coll. « Histoire », Rennes, 2008

30. CIFM, op. cit. 23 , n ${ }^{\circ} 105$, p. 108-109.

31. Estampages et frottis conservés aux Arch. dép. Vienne, 16 J 3 / 167.

32. On peut même se demander si ce bloc est dans sa position originelle : les graffitis semblent en effet incomplets à droite, comme s'ils s'étaient initialement poursuivis sur un autre bloc.

33. Arch. dép. Vienne, $16 \mathrm{~J} 3 / 167$.

34. Bertou 1900, p. LIX.

35. On connaît d'autres inscriptions médiévales présentant une suite de lettres dont la signification nous échappe encore, la plus célèbre de toutes étant sans doute l'inscription d'Ansquitil à Moissac ( $\mathrm{cf}$ CIFM, op. cit., 8, nº 10, p. 131-134), vers 1100 . 